\title{
An analysis of parasitic current generation in volume of fluid simulations
}

\author{
D. J. E. Harvie* M. R. Davidson ${ }^{\dagger} \quad$ M. Rudman ${ }^{\ddagger}$
}

(Received 29 November 2004, revised 17 February 2005)

\begin{abstract}
Parasitic currents are unphysical currents generated when using implementations of the Continuum Surface Force technique to model surface tension forces in multi-phase Computational Fluid Dynamics problems. We derive and validate a correlation for the magnitudes of these currents as a function of the physical and numerical parameters used in a given simulation. We find that these currents may be limited by both the inertial and viscous terms in the Navier-Stokes equations, and as observed by previous researchers, that they do not decrease in magnitude with increased mesh refinement nor decreased computational time step.
\end{abstract}

${ }^{*}$ Dept. Chemical \& Biomolecular Engineering, University of Melbourne, Australia. mailto:daltonh@unimelb.edu.au

${ }^{\dagger}$ Dept. Chemical \& Biomolecular Engineering, University of Melbourne, Australia.

${ }^{\ddagger}$ CSIRO, Manufacturing \& Infrastructure Technology, Melbourne, Australia.

See http://anziamj.austms.org.au/V46/CTAC2004/Har1 for this article, (C) Austral. Mathematical Soc. 2005. Published April 19, 2005. ISSN 1446-8735 


\section{Contents}

1 Introduction

C134

2 The CSF method

C136

3 The origin of parasitic currents

C138

4 Parasitic current correlation

C139

5 Numerical experiments

C142

6 Discussion

C145

7 Conclusions

C148

References

C148

\section{Introduction}

Since its development over one decade ago, the Continuum Surface Force (CSF) method has arguably become the pre-eminent tool used to model surface tension effects in Eulerian based Computational Fluid Dynamics (CFD) codes [8]. The CSF model simulates the discrete stress change that occurs at an interface due to capillary effects by a body force which acts throughout a small but finite fluid region surrounding the interface. The magnitude and direction of the force are chosen so that in the limit of this region becoming infinitely small, the correct capillary induced stress change at the interface is recovered.

Although the CSF model has been successfully employed to model a large variety of flow problems involving interfaces using various CFD methods (for example, level-set, Volume of Fluid), its ability to model flows which are 
dominated by surface tension effects is limited by the existence of so-called 'parasitic' currents. Parasitic currents are unphysical currents generated in fluid regions adjacent to an interface by local variations in the CSF body force. Their magnitude generally increases with increasing capillary strength, and may become so large as to affect the prediction of flow field velocities, or in more extreme circumstances, cause complete breakup of an interface.

Although the presence of parasitic currents has been widely observed by researchers since the conception of the CSF method [2], to date there has been little quantitative analysis of their magnitude. Lafaurie et al. [4], who coined the term 'parasitic', suggested that the dimensionless magnitude of these currents scaled with the inverse of the capillary number and supported this observation with a small series of numerical experiments. They noted however that there was also some dependence of the current magnitude on the Reynolds number as currents tended to become stronger and exhibit greater fluctuations as the Reynolds number of the system was increased. Later, Scardovelli \& Zaleski [8] proposed that the large currents observed by some researchers during high Reynolds and low Weber number computations may occur because of the dominance of surface tension relative to inertial forces, but this effect was not quantified. As we shall see however, all of these observations are consistent with the results of this study. Note that amongst others, Renardy \& Renardy [6], Meier, Yadigaroglu \& Smith [5] and Jamet, Torres \& Brackbill [3] all presented variations on the CSF technique aimed at reducing the magnitudes of parasitic currents.

Our purpose is to determine a correlation for the maximum parasitic current magnitude as a function of the physical and numerical parameters used in a simulation. This is achieved by performing an order of magnitude analysis on the discretised Navier-Stokes equations with the CSF force included. The correlation is validated by comparing predicted velocity magnitudes against those obtained from a variety of numerical experiments, performed using the Volume of Fluid (VOF) algorithm due to Rudman [7]. 


\section{The CSF method}

In this section we briefly review the CSF method. Details of the particular numerical implementation of this method used in this study can be found in $[7]$.

The Navier-Stokes momentum equation for incompressible Newtonian flow of two immiscible liquids with body forces neglected is

$$
\frac{\partial \rho \boldsymbol{u}}{\partial t}+\boldsymbol{\nabla} \cdot \rho \boldsymbol{u} \boldsymbol{u}=-\boldsymbol{\nabla} p+\frac{1}{\mathrm{We}} \kappa_{\mathrm{s}} \hat{\boldsymbol{n}}_{\mathrm{s}} \delta\left(\boldsymbol{x}-\boldsymbol{x}_{\mathrm{s}}\right)+\frac{1}{\mathrm{Re}} \boldsymbol{\nabla} \cdot \mu\left[\boldsymbol{\nabla} \boldsymbol{u}+(\boldsymbol{\nabla} \boldsymbol{u})^{\mathrm{\top}}\right] .
$$

This non-dimensional equation has velocity non-dimensionalised by $\bar{v}$, length by $\bar{x}$, density by the continuous phase density $\bar{\rho}_{\mathrm{c}}$, viscosity by the continuous phase viscosity $\bar{\mu}_{\mathrm{c}}$, pressure by $\bar{\rho}_{\mathrm{c}} \bar{v}^{2}$ and time by $\bar{x} / \bar{v}$. Note that variables having over-bars (for example, $\bar{x}$ ) have dimensions. In this study we refer to one immiscible fluid as the 'continuous' phase and the other immiscible fluid as the 'disperse' phase. These terms have been adopted for reasons of convention only - under the CSF method both fluids are treated identically and can be interchanged.

Dimensionless groups relevant to the problem are the Reynolds number $(\mathrm{Re})$, the Weber number (We) and the capillary number (Ca). The capillary number represents the ratio of viscous to surface tension forces. These numbers are defined using continuous phase properties as

$$
\mathrm{Re}=\frac{\bar{\rho}_{\mathrm{c}} \bar{v} \bar{x}}{\bar{\mu}_{\mathrm{c}}}, \quad \mathrm{We}=\frac{\bar{\rho}_{\mathrm{c}} \bar{v}^{2} \bar{x}}{\bar{\sigma}} \quad \text { and } \quad \mathrm{Ca}=\frac{\mathrm{We}}{\mathrm{Re}}=\frac{\bar{v} \bar{\mu}_{\mathrm{c}}}{\bar{\sigma}},
$$

respectively.

The momentum equation given in (1) is conventional except for the second term on the right hand side. This term represents a surface tension induced stress jump which acts along any disperse-continuous phase interface. As we consider only systems for which the surface tension $(\bar{\sigma})$ is uniform, this force 
is everywhere directed normal to the interface. The direction of this force is $\hat{\boldsymbol{n}}_{\mathrm{s}}$, a unit vector which is defined only on the disperse-continuous phase interface and is directed normal to this interface and into the disperse phase. Other variables in the surface tension term include $\kappa_{\mathrm{s}}$, the signed magnitude of curvature of the interface, $\delta\left(\boldsymbol{x}-\boldsymbol{x}_{\mathrm{s}}\right)$, the Dirac delta function, and $\boldsymbol{x}_{\mathrm{s}}$, which specifies the location of the interface. The subscript $s$ here signifies that these variables are associated with a surface (the disperse-continuous phase interface).

The surface tension term in equation (1) is problematic to implement in a fixed or Eulerian mesh numerical scheme (especially in three dimensions) as it is non-zero only on a surface, rather than acting continuously over a volume as the other terms in the momentum equation do. The CSF technique alleviates this problem by replacing the surface force in equation (1) by a volume force which acts over a small region surrounding any disperse-continuous phase interface. Specifically under the CSF method the surface tension term in equation (1) is replaced by

$$
\frac{1}{\mathrm{We}} \frac{\rho}{\langle\rho\rangle} \kappa_{\mathrm{v}} \boldsymbol{n}_{\mathrm{v}} .
$$

The normal in (3) is defined in terms of a continuous scalar field $c$ as $\boldsymbol{n}_{\mathrm{v}}=$ $\boldsymbol{\nabla} c /\left(c_{\mathrm{d}}-c_{\mathrm{c}}\right)$ and the curvature is defined in terms of the new interface normal as $\kappa_{\mathrm{v}}=-\boldsymbol{\nabla} \cdot \hat{\boldsymbol{n}}_{\mathrm{v}}$ where $\hat{\boldsymbol{n}}_{\mathrm{v}}=\boldsymbol{n}_{\mathrm{v}} /\left|\boldsymbol{n}_{\mathrm{v}}\right|$. Thus, both the normal vector and curvature used in equation (3) are now defined consistently throughout the fluid volume rather than just on a surface. In the present implementation, $c$ is taken to be the VOF function $\phi$ (that is, the volume fraction of the disperse phase) so that $\left(c_{\mathrm{d}}-c_{\mathrm{c}}\right)=1$ and $\boldsymbol{n}_{\mathrm{v}}=\boldsymbol{\nabla} \phi$. As $\phi$ is constant within each fluid phase, the normal $\boldsymbol{n}_{\mathrm{v}}$, and thus the surface force, is non-zero only within a small region surrounding any disperse-continuous phase interface.

The surface force defined in equation (3) includes a density correction suggested by Brackbill, Kothe \& Zemach [2] for modeling systems where the phases have unequal densities. Here $\rho=\phi\left(\rho_{\mathrm{d}}-1\right)+1$ is the local nondimensional density and $\langle\rho\rangle=\left(1+\rho_{\mathrm{d}}\right) / 2$ is the average non-dimensional 
density of the two phases. Including these two variables in (3) has the effect of weighting the surface tension force towards regions of higher density, thus tending to produce more uniform fluid accelerations across the width of the disperse-continuous phase interface region.

\section{$3 \quad$ The origin of parasitic currents}

To illustrate how parasitic currents develop, we consider the forces that act on a two-dimensional planar droplet suspended in a continuous phase, all within a zero gravity environment.

Physically, once equilibrium is established in such a system the droplet would assume the shape of a circle and all fluid velocities would be zero. Referring back to the momentum equation (1) we see that all terms involving velocities in this system would be zero, the curvature around the interface of the droplet would be uniform, the interface normal would be directed everywhere in the radial direction, and the non-dimensional pressure would be uniform within and outside the droplet but undergo a step change of magnitude $1 /(a \mathrm{We})$ across the interface. Here $a$ is the non-dimensional radius of the droplet.

We now consider the same droplet, but in terms of an 'ideal' voF method. Under the VOF method, the location of the disperse phase is determined by the scalar volume fraction $\phi$. Employing a two-dimensional cylindrical coordinate system $(r, \theta)$, under this ideal numerical method $\phi$ would be a function of $r$ only, and would be equal to one for $r \ll a$, zero for $r \gg a$, and would vary monotonically between these two values over the interface region where $r \approx a$. Using the definitions given in the previous section, $\hat{\boldsymbol{n}_{\mathrm{v}}}$ would be a unit vector defined within the interface region pointing inwards in the radial direction, $\kappa_{\mathrm{v}}=1 / r$ a function of $r$ only, and the density, which is a function of $\phi$, also a function of $r$ only. Thus, the entire surface volume interface force given by equation (3) would be a vector directed in the radial direction, 
with a magnitude that is a function of $r$ only. Such a force is irrotational and so can be represented as the gradient of a scalar field. Referring back to the momentum equation (1) but with the volume surface tension force now substituted for the surface based force, we see that the long time for solution for this system under this ideal voF method would be for the surface tension force to be precisely balanced by the pressure gradient term, with all velocity dependent terms, and thus velocities, being zero.

A real vof numerical implementation of this system differs from this ideal implementation in that $\phi$, which when discretised represents the volume fraction integrated over the dimensions of a computational mesh cell, varies by a small amount in the $\theta$ direction as well as in the $r$ direction. This results in $\hat{\boldsymbol{n}}_{\mathrm{v}}$ being not precisely directed in the radial direction, $\kappa_{\mathrm{v}}$ varying slightly in the $\theta$ direction as well as the $r$ direction, $\rho$ also having $\theta$ direction variations, and the complete interface volume force given by equation (3) having an 'erroneous' rotational component. Referring again to the momentum equation (1) but with the volume force again replacing the surface based surface tension force, see that the 'erroneous' rotational component of the surface tension force cannot be balanced by the irrotational pressure gradient term, so must instead be balanced by one or more of the three other velocity dependent terms. As these velocity terms (inertial transient, inertial advection and viscous) all require non-zero velocities if they themselves are to be non-zero, spurious currents develop.

\section{Parasitic current correlation}

To develop a correlation for the magnitude of these currents, we employ the conservative assumption that the erroneous rotational component of the surface tension force is balanced by one of the velocity dependent terms in the momentum equation; the specific velocity term being the one which balances the surface tension error with the lowest current magnitudes. 
Consider first a balance between the viscous and surface tension terms. By equating the order of these we find

$$
\mathcal{O}\left(\frac{1}{\mathrm{We}} \frac{\rho}{\langle\rho\rangle} \kappa_{\mathrm{v}} \boldsymbol{n}_{\mathrm{v}}\right)=\mathcal{O}\left(\frac{1}{\operatorname{Re}} \boldsymbol{\nabla} \cdot \mu\left[\boldsymbol{\nabla} \boldsymbol{u}_{\mathrm{P}}+\left(\boldsymbol{\nabla} \boldsymbol{u}_{\mathrm{P}}\right)^{\top}\right]\right),
$$

where $\boldsymbol{u}_{\mathrm{P}}$ represents the parasitic velocity field. From the definitions presented in Section 2, $\kappa_{\mathrm{v}}$ is calculated as the divergence of a unit vector. In the numerical implementation, this divergence is approximated as the difference of an order one quantity over a mesh dimension of length $h$. Thus, we would expect the maximum magnitude of error in $\kappa_{\mathrm{v}}$ to scale as $1 / h$. Similarly, $\boldsymbol{n}_{\mathrm{v}}$ is defined as the gradient of the order one quantity $\phi$. We would therefore expect the maximum error in this normal magnitude to also scale as $1 / h$.

On the right hand side of equation (4), we have the divergence and gradient of the parasitic current velocity field. Parasitic currents are observed to circulate over length scales of similar magnitude to the finite interface region width, a dimension which is kept proportional to the mesh dimension $h$ in this study. Thus, we would expect the viscous term to scale as $\mu U_{\mathrm{P}} /\left(\operatorname{Re} h^{2}\right)$ where $U_{\mathrm{P}}$ is a measure of the parasitic current magnitude. Employing these assumptions in equation (4) we find for the parasitic current magnitude

$$
U_{\mathrm{P}} \propto \frac{\operatorname{Re}}{\operatorname{We}} \frac{\rho}{\langle\rho\rangle} \frac{1}{\mu} .
$$

The local viscosity $\mu$ is defined in a similar fashion to $\rho$ as a function of $\phi$. However, in the numerical discretisation of the momentum equation, $\rho$ and $\mu$ are not evaluated at the same position so that near the interface the ratio $\rho / \mu$ can vary widely from location to location. Thus, the maximum parasitic velocities derived from a balance between the surface tension and viscous terms will scale as $U_{\mathrm{V}}$, where

$$
U_{\mathrm{V}}=\frac{2 \max \left(\rho_{\mathrm{d}}, 1\right)}{\mathrm{Ca}\left(1+\rho_{\mathrm{d}}\right) \min \left(\mu_{\mathrm{d}}, 1\right)}
$$


and $\mathrm{Ca}$ is the capillary number defined in Section 2.

We now turn our attention to the inertial advection term. Equating the order of magnitude of this term with that of the surface tension term we find

$$
\mathcal{O}\left(\frac{1}{\mathrm{We}} \frac{\rho}{\langle\rho\rangle} \kappa_{\mathrm{v}} \boldsymbol{n}_{\mathrm{v}}\right)=\mathcal{O}\left(\boldsymbol{\nabla} \cdot \rho \boldsymbol{u}_{\mathrm{P}} \boldsymbol{u}_{\mathrm{P}}\right),
$$

where $\boldsymbol{u}_{\mathrm{P}}$ again represents the generated parasitic current velocity field. On the left of this equation, we can employ the same scaling assumptions as used above for the magnitudes of error in the normal and curvature variables. On the right, we again note that observed parasitic currents circulate over dimensions that scale with the mesh size $h$, so that the entire advection term scales as $\rho U_{\mathrm{P}}^{2} / h$. Again $U_{\mathrm{P}}$ is a measure of the parasitic current magnitude.

In the numerical discretisation of the momentum equation (1), the density used in the surface tension volume force term and that used in the inertial advection term are evaluated at slightly different locations. This would suggest that the densities on the right and left of equation (7) cannot be canceled. However, as the density used in the advection term is calculated from adjacent mass cell centred values, this is a reasonable assumption and one which is supported by the numerical experiments presented later. Thus, we find that a balance between the inertial advection and surface tension terms leads to the maximum parasitic current magnitude scaling as $U_{\mathrm{A}}$, where

$$
U_{\mathrm{A}}=\sqrt{\frac{2}{\mathrm{We}\left(1+\rho_{\mathrm{d}}\right) h}} .
$$

Finally we consider a balance between the inertial transient and surface tension terms. Equating these terms in an order of magnitude sense we find

$$
\mathcal{O}\left(\frac{1}{\mathrm{We}} \frac{\rho}{\langle\rho\rangle} \kappa_{\mathrm{v}} \boldsymbol{n}_{\mathrm{v}}\right)=\mathcal{O}\left(\frac{\partial \rho \boldsymbol{u}_{\mathrm{P}}}{\partial t}\right) .
$$

On the left of this equation we can employ the same scalings as used previously. On the right, the inertial transient term will scale as $U_{\mathrm{P}} \rho / t$ where $t$ is 
the maximum time that any particular mesh cell has contained an interface region. If the interface regions are stationary (as in the numerical experiments presented next) $t$ is simply the time elapsed since the beginning of the test. Since the density used in the inertial transient term is evaluated at the same location as the density in the surface tension term, we can cancel them from both sides of equation (9) to find that a balance between the transient inertial and surface tension terms gives parasitic currents that scale as $U_{\mathrm{T}}$, where

$$
U_{\mathrm{T}}=\frac{2 t}{\mathrm{We}\left(1+\rho_{\mathrm{d}}\right) h^{2}} .
$$

Using our conservative assumption that in a given problem the erroneous surface tension force will be limited by the velocity term which produces the smallest parasitic current magnitude, we can combine equations (6), and (10) to form a single correlation for the maximum parasitic current magnitude,

$$
U_{\mathrm{P}}=\min \left(a_{\mathrm{T}} U_{\mathrm{T}}, a_{\mathrm{A}} U_{\mathrm{A}}, a_{\mathrm{V}} U_{\mathrm{V}}\right),
$$

where $a_{\mathrm{T}}, a_{\mathrm{A}}$ and $a_{\mathrm{V}}$ are constants specific to the particular numerical implementation of the CSF technique.

\section{$5 \quad$ Numerical experiments}

We now present results of numerical experiments performed using the Rudman [7] implementation of the CSF technique in which we measure maximum parasitic current magnitudes as a function of various physical and numerical parameters. The purpose of these experiments is to validate the correlation developed in the previous section, and at the same time, estimate the magnitude of the constants $a_{\mathrm{T}}, a_{\mathrm{A}}$ and $a_{\mathrm{V}}$ valid for this particular CSF implementation.

The model problem we chose is that of a two dimensional planar droplet, of radius $1 / 2$, centred at the point $(1,1)$ and in a total domain of size $2 \times 2$. 
TABLE 1: Physical and numerical parameters varied in the numerical experiments and the ranges over which they were varied.

\begin{tabular}{cl} 
Parameter & Range \\
\hline$t$ & $1 \rightarrow 10$ \\
$h$ & $1 / 8 \rightarrow 1 / 256$ \\
$\mathrm{We}$ & $10^{-6} \rightarrow 10^{4}$ \\
$\mathrm{Ca}$ & $10^{-5} \rightarrow 10^{4}$ \\
$\rho_{\mathrm{d}}$ & $10^{-4} \rightarrow 10^{4}$ \\
$\mu_{\mathrm{d}}$ & $10^{-4} \rightarrow 10^{4}$
\end{tabular}

Computational boundaries were modeled as non-slip and the domain was discretised using a uniform, square mesh. A kernel radius of 3.5 fine mesh cell dimensions was used when calculating $\boldsymbol{n}_{\mathrm{v}}$ and $\kappa_{\mathrm{v}}$ [7]. Note that as no body force acts on this droplet, its equilibrium position should be the same as its initial position and all velocities should be zero.

Table 1 shows the parameters that are varied in the numerical experiments. The cell dimension $h$ here is taken to be the side length of the coarse momentum and mass cells. Note that in total over 170 numerical experiments were performed, and the magnitude of currents measured in the tests was found to vary over 8 orders of magnitude. Multiple results at different times were taken for some tests to validate the form of the transient inertial term in equation (11).

To determine the constants in equation (11), we calculated the difference between the current magnitude predicted by the correlation and that measured experimentally for each test, averaged these differences over all tests to find an average error, and using a regression analysis, found values of $a_{\mathrm{T}}$, $a_{\mathrm{A}}$ and $a_{\mathrm{V}}$ which minimised this error. A comparison of the resulting correlation and experimental values is shown in Figure 1, along with the values found for the constants. As shown, the correlation predicts the order of magnitude of the parasitic currents accurately as they vary from less than $10^{-7}$ 


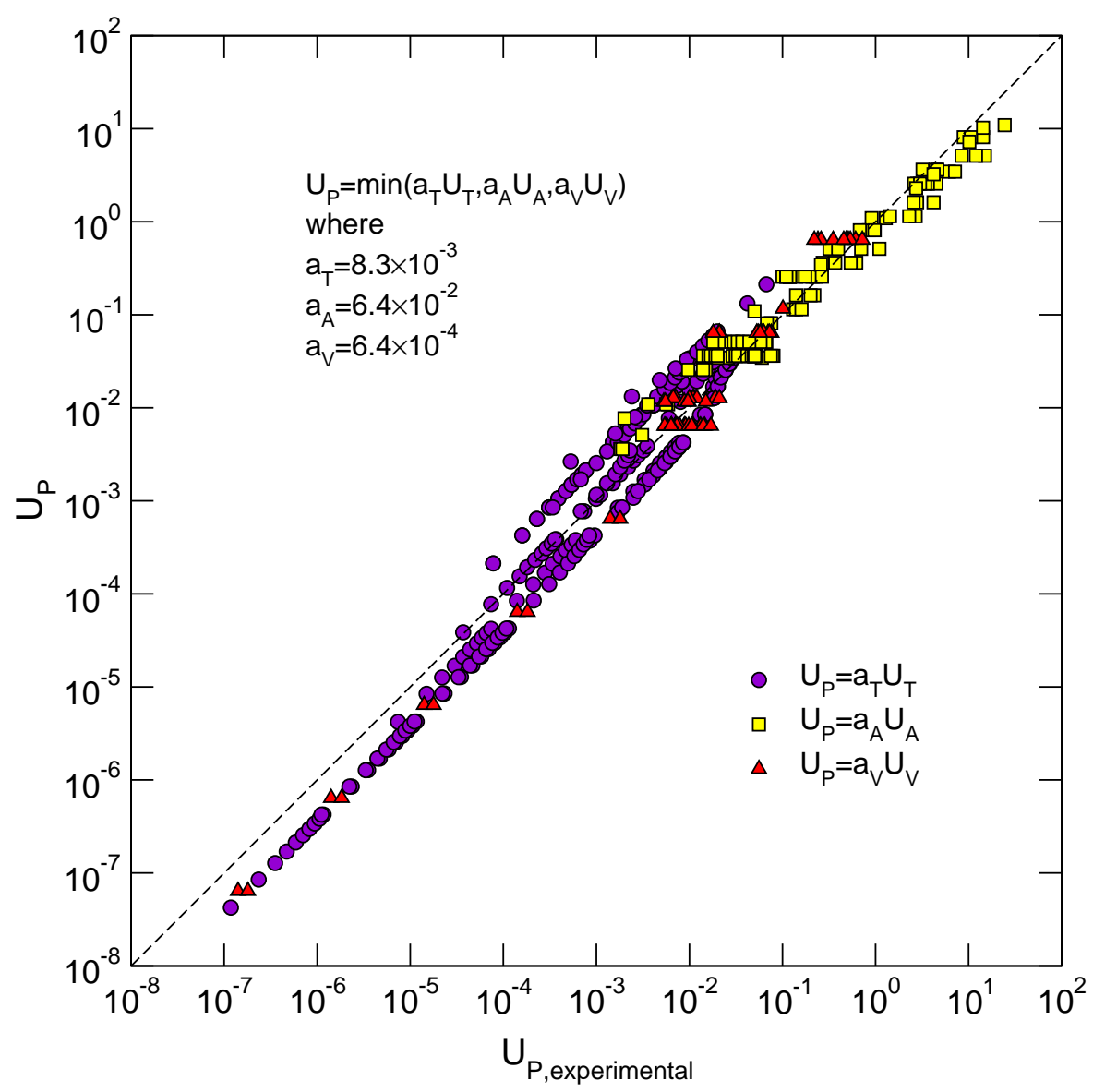

FiguRE 1: Parasitic current magnitudes predicted by the correlation $\left(U_{\mathrm{P}}\right)$ plotted against those measured in the numerical experiments $\left(U_{\mathrm{P} \text {,experimental }}\right)$. The values of $a_{\mathrm{T}}, a_{\mathrm{A}}$ and $a_{\mathrm{V}}$ found from the regression analysis and used to determine $U_{\mathrm{P}}$ are listed. The particular term of the correlation that limited the current magnitude is indicated by the symbol type. 
to greater than $10^{1}$.

\section{Discussion}

A significant conclusion that can be drawn from the results of Figure 1 is that parasitic currents can be limited by the inertial terms in the NavierStokes equations as well as by the viscous term. Currents will generally only be limited by the transient inertial term $\left(a_{\mathrm{T}} U_{\mathrm{T}}\right)$ if the disperse phase field continuously evolves with time. If the disperse phase field remains stationary then $t$ will eventually become large and the inertial advection or viscous terms will take over limiting the maximum current magnitude.

Currents limited by the inertial advection term $\left(a_{\mathrm{A}} U_{\mathrm{A}}\right)$ are interesting as they decrease as the Weber number of the simulation increases, but increase as $h$ is decreased. This latter observation shows that as the mesh is refined, the magnitude of generated currents actually increases. This increase will eventually stop with decreasing $h$; however, as the current magnitude limited by the viscous term is independent of mesh size so that eventually $a_{\mathrm{A}} U_{\mathrm{A}}>$ $a_{\mathrm{V}} U_{\mathrm{V}}$ and the viscous term will begin to limit the current magnitude. Note that the computational time step does not affect the current magnitude.

Previous investigators have stated that parasitic current magnitudes generally scale as $1 / \mathrm{Ca}$. If the viscosities and densities of the phases are equal, and We is low so that inertial effects are not relevant, then the parasitic currents become limited by the viscous term and equation (11) reduces to $U_{\mathrm{P}}=a_{\mathrm{V}} / \mathrm{Ca}$. This is the same relationship given by Lafaurie [4], who had an equivalent $a_{\mathrm{V}}$ of approximately $1 \times 10^{-2}$ for their surface tension implementation. The lower value of $a_{\mathrm{V}}=6.4 \times 10^{-4}$ determined in this study indicates the increased accuracy of the Rudman CSF implementation over Lafaurie et al.'s earlier method. Brackbill, Kothe \& Zemach [1] proposed an alternative CSF method in which the surface tension force is decomposed into irrotational and rotational fields. They report a value of $a_{\mathrm{V}} \approx 6 \times 10^{-4}$ 


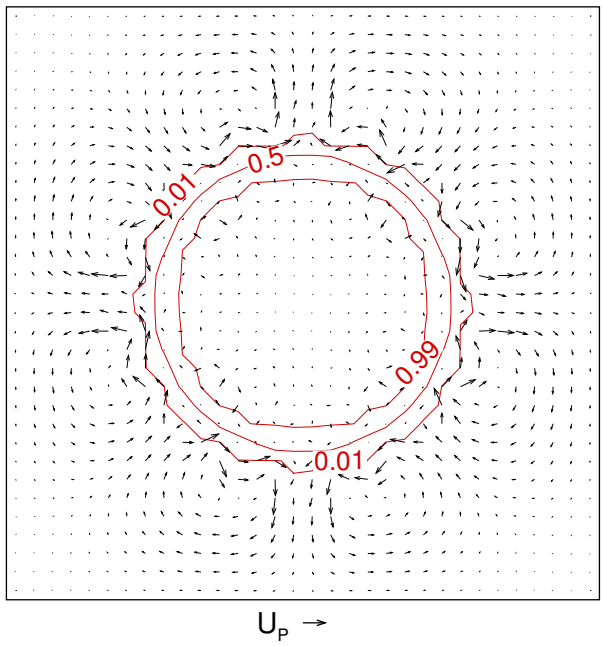

(a) $\mathrm{Ca}=2.13 \times 10^{-4}, \mathrm{We}=1 . \times 10^{-3}$ and $U_{\mathrm{P}}=a_{\mathrm{V}} U_{\mathrm{V}}=3.01$.

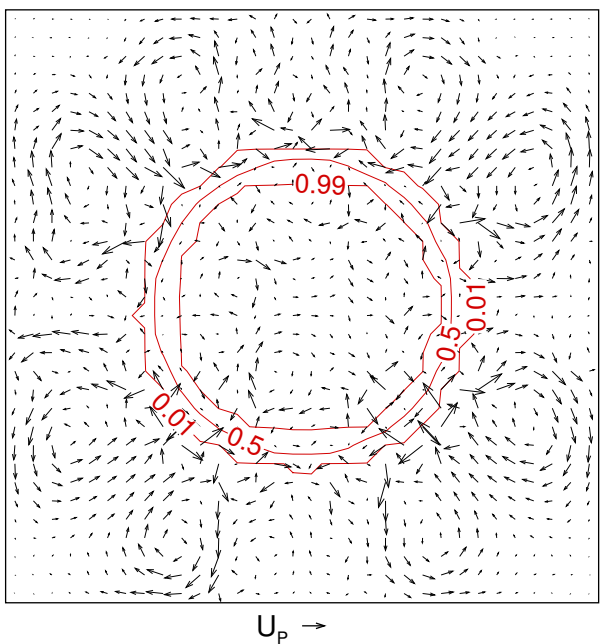

(b) $\mathrm{Ca}=1 . \times 10^{-5}, \mathrm{We}=1 . \times 10^{-3}$ and $U_{\mathrm{P}}=a_{\mathrm{A}} U_{\mathrm{A}}=3.01$.

Figure 2: Parasitic currents calculated at $t=4$ for a planar two dimensional droplet. The Weber and capillary numbers have been chosen so that $U_{\mathrm{P}}$ is the same for both frames, however in Frame $2\left(\right.$ a) $U_{\mathrm{P}}$ is limited by the viscous term while in Frame 2(b) it is limited by the inertial advection term. $h=1 / 16$ and $\rho_{\mathrm{d}}=\mu_{\mathrm{d}}=1 \mathrm{in}$ both cases. The contours are lines of constant volume fraction $\phi$. 
which is almost the same as the value determined here.

Figure 2 shows example velocity fields calculated for the model planar drop problem: In one case the currents are limited by the viscous term (Frame 2(a)) while in the other they are limited by the inertial advection term (Frame 2(b)). The Reynolds and Weber numbers in these cases were chosen so that $U_{\mathrm{P}}$ was equal in each. The velocity vectors for the viscous limited case were found to be quite time independent throughout the computation, and as shown, were largest near the interface between the two phases. In the viscous limited case the droplet remained stationary. In the inertially limited case the parasitic currents tended to spread across the entire computational domain, eventually moving the droplet away from the centre and along a relatively random trajectory. While the form of the velocity field changed with time in the inertia limited case, the maximum velocity magnitudes remained constant. This is because the 'erroneous' CSF force acts on the velocity field in directions which are independent of the velocity direction: While the force may increase parasitic currents at one location, at another it may oppose them.

While parasitic current generation may be small if the disperse phase field changes continuously with time (as is the case in many practical problems), or may be confined to only small regions of a computational domain, the developed correlation shows that current generation can be large for many physically relevant systems. What implications these currents have on the accuracy of other simulation results, such as droplet shapes, drag coefficients and pressure fields, has not been analysed. The CSF method has been used to accurately predict droplet shapes and terminal rise/fall velocities in regimes where parasitic currents are seen to be significant. However, in some situations the presence of parasitic currents is known to invalidate simulation results, particularly when breakup of the disperse-continuous phase interface results. For these reasons, more research is required to assess the effect that parasitic currents have on simulation accuracy, particularly with regard to disperse phase interface geometry and stress fields. 


\section{Conclusions}

A correlation describing the magnitudes of parasitic currents that develop during simulations using the CSF surface tension technique has been derived. This correlation has been validated using a series of numerical experiments performed using a VOF algorithm. The correlation shows that when a system is inertia dominated, the magnitude of generated currents can increase with increasing mesh refinement. When a system is viscosity dominated, the currents scale with the inverse of the capillary number as has previously been reported. Current magnitudes are not affected by the computational time step. Future work will assess what implications the presence of parasitic currents has on the accuracy of CSF simulations beyond simply inaccuracies in the observed velocity field.

Acknowledgment: This research was supported by the Australian Research Council Linkage Grants Scheme.

\section{References}

[1] J. U. Brackbill, D. Juric, D. Torres, and E. Kallman. Dynamic modelling of microgravity flow. In Fourth Microgravity Fluid Physics and Transport Phenomena Conference, pages 584-589, Cleveland, Ohio, USA, Aug. 12-14 1998. National Centre for Microgravity Research. C145

[2] J. U. Brackbill, D. B. Kothe, and C. Zemach. A continuum method for modelling surface tension. Journal of Computational Physics, 100:335-354, 1992. C135, C137

[3] D. Jamet, D. Torres, and J. U. Brackbill. On the theory and computation of surface tension: The elimination of parasitic currents 
through energy conservation in the second-gradient method. Journal of Computational Physics, 182:262-276, 2002. C135

[4] B. Lafaurie, C. Nardone, R. Scardovelli, S. Zaleski, and G. Zanetti. Modelling merging and fragmentation in multiphase flows with SURFER. Journal of Computational Physics, 113:134-147, 1994. C135, C145

[5] M. Meier, G. Yadigaroglu, and B. L. Smith. A novel technique for including surface tension in plic-vof methods. Eur. J. Mech. B/Fluids, 21:61-73, 2002. C135

[6] Y. Renardy and M. Renardy. Prost: A parabolic reconstruction of surface tension for the volume-of-fluid method. Journal of Computational Physics, 183:400-421, 2002. C135

[7] M. Rudman. A volume-tracking method for incompressible multifluid flows with large density variations. International Journal for Numerical Methods in Fluids, 28:357-378, 1998. C135, C136, C142, C143

[8] R. Scardovelli and S. Zaleski. direct numerical simulation of free-surface and interfacial flow. Annual Review of Fluid Mechanics, 31:567-603, 1999. C134, C135 